\title{
Fetal Mediastinal Mass Associated with Arrhythmia: Artifact and Casual Finding in Ultrasound Diagnosis
}

\author{
${ }^{1}$ Olga Rosales-Aedo, ${ }^{2}$ Margarita Alvarez-de-la-Rosa, ${ }^{3}$ Ana I Padilla, ${ }^{4} J u a n$ M Troyano
}

\author{
ABSTRACT \\ Aim: We aim to report a case of an enlarged fetal thymus \\ causing arrhythmia.
}

Background: Fetal mediastinal masses may be clinically asymptomatic or cause hemodynamically significant alterations, heart failure, arrhythmias, and sudden cardiac death.

Case description: We present a case of fetal mediastinal mass associated with an arrhythmia. A 29-year-old primigravida, with no previous medical history, was referred to our center due to suspicion of fetal arrhythmia detected in a routine control in week 34. A thymus of hyperplasic characteristics was suspected in the ultrasound evaluation. Pregnancy concluded uneventfully with favorable neonatal outcome.

Conclusion: The fetal thymus is a structure that usually goes unnoticed during the process of prenatal diagnosis, and when it presents alterations in its morphology, can lead to confusion.

Clinical significance: The fetal thymus can be physiologically enlarged and cause fetal arrhythmias without an adverse clinical outcome.

Keywords: Arrhythmia, Echocardiography, Mediastinum, Prenatal diagnosis, Thymus.

How to cite this article: Rosales-Aedo O, Alvarez-de-la-Rosa M, Padilla Al, Troyano JM. Fetal Mediastinal Mass Associated with Arrhythmia: Artifact and Casual Finding in Ultrasound Diagnosis. Donald School J Ultrasound Obstet Gynecol 2018;12(4):258-261.

Source of support: Nil

Conflict of interest: None

\section{BACKGROUND}

The thymus originates bilaterally from the third and fourth branchial arches. ${ }^{1}$ Its development begins in the sixth week of gestation until weeks 14-16; after that, the

${ }^{1}$ Resident, ${ }^{2,3}$ Associate Professor, ${ }^{4}$ Professor

${ }^{1-4}$ Universidad de La Laguna, Departamento de Obstetricia y Ginecología, Hospital Universitario de Canarias, Santa Cruz de Tenerife, Spain

Corresponding Author: Margarita Alvarez-de-la-Rosa, Associate Professor, Universidad de La Laguna, Departamento de Obstetricia y Ginecología, Hospital Universitario de Canarias, Santa Cruz de Tenerife, Spain, Phone: +34 922 678000, e-mail: malvrod@ull.edu.es thymus grows rapidly. ${ }^{2}$ In fetal ultrasound, the thymus is better visualized in the axial view, ventral to the great vessels and dorsal to the sternum or clavicles. ${ }^{3}$ It presents as a homogeneous structure in the anterior mediastinum with an ovoid or pyramidal shape, but it can be difficult to visualize in its entirety due to its position behind the sternum and the ribs. ${ }^{3}$

Fetal mediastinal masses may be clinically asymptomatic or cause hemodynamically significant alterations, heart failure, arrhythmias, and sudden cardiac death, ${ }^{4}$ however, most arrhythmias caused by mediastinal masses are attributed to the presence of cardiac rhabdomyomas. There is no certainty about the presence of thymic hyperplasia leading to autoimmune diseases during intrauterine development. ${ }^{5}$ On the contrary, it is well known that, over the years, hypoplasia or fetal thymic aplasia has been related as one of the forms of manifestation of various clinical scenarios, such as the deletion of chromosome 22q11.2, chondrodysplasia punctata, Ellis-van Creveld syndrome and after prenatal exposure to agents such as ethanol and the human immunodeficiency virus; however, there is little reported in the literature about the increase in the size of said gland during fetal development, ${ }^{6}$ and currently published studies emphasize the need for additional functional analysis. ${ }^{7}$

The normal size of the fetal thymus has been tabulated with different variants for its measurement: for example, by using the anteroposterior thickness by Felker et al. ${ }^{8}$ and by the perimeter of Zalel et al. ${ }^{3}$ The transverse thymic diameter progressively increases as the gestational age progresses, ${ }^{9}$ without finding statistically significant differences between the size of the fetal thymus in female or male fetuses. ${ }^{10}$ To make the correct diagnosis of a fetal anomaly, careful evaluation of the location and sonographic characteristics of the thymus is essential. In our review, we present a case of fetal mediastinal mass associated with an arrhythmia with favorable neonatal outcome. To the best of our knowledge, there are no publications on arrhythmias concurrent with masses originated by the fetal thymus.

\section{CASE DESCRIPTION}

We present a case report of a 29-year-old primigravida, with no previous medical history. The patient was 
referred to our center for ultrasound evaluation due to suspicion of fetal arrhythmia detected in a routine control in week 34. The pregnancy had been of physiological course and showed a combined first-trimester screening risk of 1/14649 and a nuchal translucency of $1.20 \mathrm{~mm}$. The patient had no family history of medical interest nor consanguinity and also denied the use of toxics or drugs.

During the abdominal ultrasound, the fetus had a cephalic position, biometrics in the 70th percentile, normally inserted placenta, normal amount of amniotic fluid, and physiological Doppler pulsatility index of the umbilical artery.

Fetal echocardiogram showed a normal-sized fetal heart, with situs solitus and no pericardial effusion. During the examination, the presence of self-limited episodes of fetal supraventricular arrhythmia was confirmed. In the four-chamber view, the outflows of the great vessels and both vascular arches were normal; except a discrete irregularity at foramen ovale level that had no hemodynamic repercussion. Incidentally, a homogeneous hypoechoic right para cardiac image was observed,

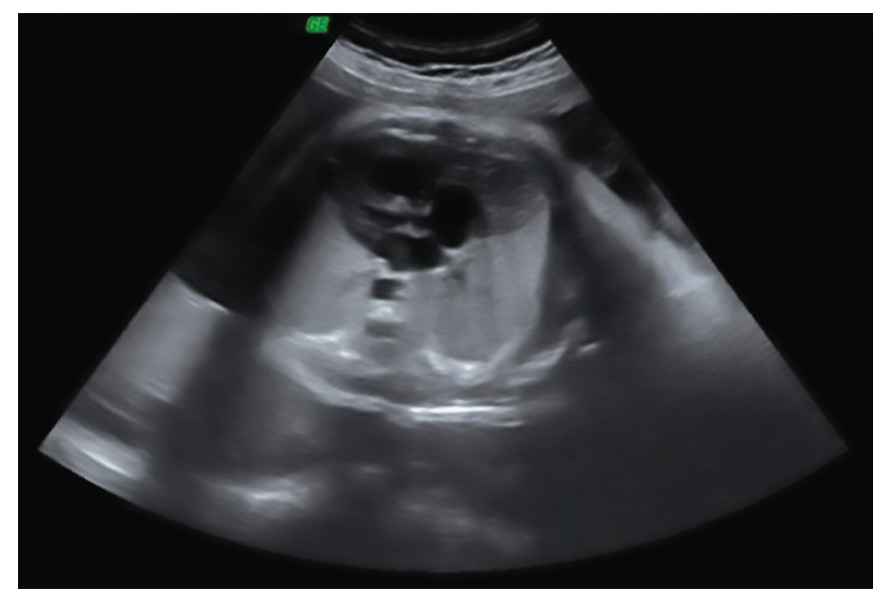

Fig. 1: Right paracardiac image is visualized, hypoechoic in relation to the adjacent lung parenchyma, at 35 weeks and 2 days of gestational age

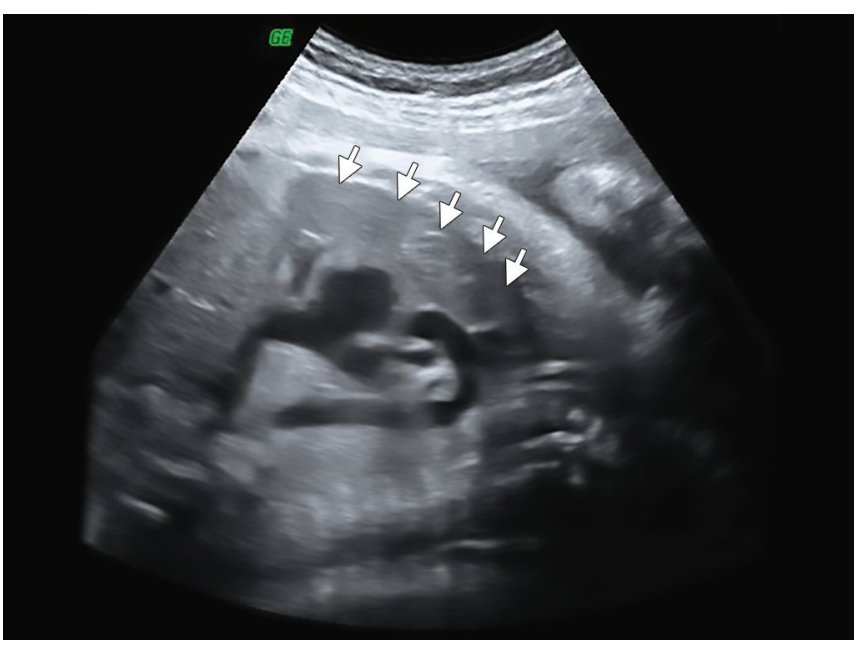

Fig. 3: Thymic image suggestive of hyperplastic characteristics well delimited and with regular vascularization, 22 × 37 $\mathrm{mm}$ in size and that remained stable during the three ultrasound tests performed between 34 weeks and 4 days and 36 weeks and 4 days of gestation. A thymus of hyperplasic characteristics was suspected (Figs 1 to 3 ). In addition, follow-up was carried out by means of a cardiotocographic (CTG) recording that started from the moment of diagnosis, carrying out a total of 9 controls in the fetal physiopathology unit. Fetal monitoring was performed with the presence of a reassuring fetal pattern, normal basal fetal heart rate, moderated variability, with accelerations, no decelerations, and presence of fetal arrhythmia without associated uterine dynamics registered or perceived by the patient, during 6 weeks of follow-up (Fig. 4).

Finally, labor started spontaneously at 40 weeks and four days of gestation. During the dilation, the patient received epidural analgesia and oxytocin in intravenous infusion at $0.3 \mathrm{~mL} /$ hour due to hypotonia. Fetal arrhythmia persisted during intrapartum monitoring. Forceps delivery was performed due to the risk of fetal distress and a male newborn of $4100 \mathrm{~g}$, 93rd percentile, Apgar 9/9 was obtained.

Postnatally, thoracic radiography, echocardiography, and electrocardiogram were performed, concluding as final diagnostic finding an aneurysmal oval fossa and

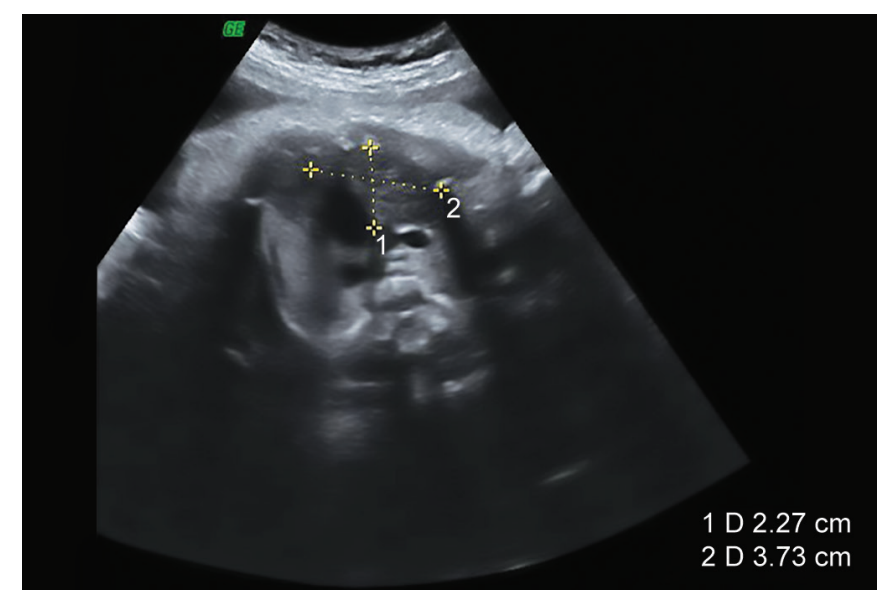

Fig. 2: $22 \times 37 \mathrm{~mm}$ image visualized in the superior mediastinum in front of the exit of the great vessels, at 35 weeks and 2 days

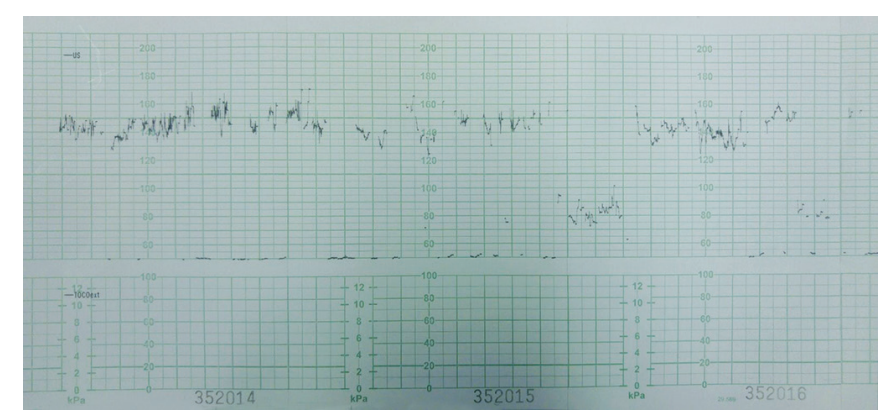

Fig. 4: Fetal arrhythmia in the electronic fetal monitor, with 40 weeks and 4 days 
the absence of arrhythmia in the newborn, who did not require hospital admission. An enlarged thymic gland was observed in the postnatal ultrasound, which in later controls decreased to a normal morphology at 4 years and 11 months of life. The child thrives normally.

\section{DISCUSSION}

The fetal thymus can be the object of errors and artifacts associated with ultrasound images and lead to overdiagnosis and overtreatment. The association of physiological thymic hyperplasia with fetal arrhythmia has not previously been described.

A relationship has been found between maternal obesity and increased fetal thymic gland, which may be related to immunological abnormalities in these fetuses. ${ }^{11}$ On the other hand, the decrease in the size of the fetal thymus has been described in fetuses affected with Down syndrome. ${ }^{12}$ Also, variations in the size of the fetal thymus have been described in cases of impaired immunity or infection. ${ }^{9}$

There are standards that have been described in the ultrasound parameters for the correct visualization and measurement of the fetal thymus, known as Thy Box. ${ }^{13}$ Confirmation of the location of this structure in the anterior mediastinum can be done by turning the transducer in the sagittal plane. The echogenicity of the thymus in relation to the pulmonary fields may change during pregnancy, being hyperechogenic or isoechogenic to the lungs prior to 27 weeks of gestation and hypoechoic with respect to the lungs after 27 weeks of gestation, ${ }^{8}$ as shown in the images of this clinical case.

As already mentioned, ultrasound measurements of the thymus are similar in male and female fetuses and present common ultrasound measurements that vary with gestational age and fetal biometry, with no significant differences in large fetuses for gestational age. ${ }^{10}$ We believe that the enlargement of the fetal thymus is not attributable to the macrosomy in this case. We hypothesize that the mass effect on the pericardium could be the cause of the fetal arrhythmia, as previously published..$^{14}$ Nevertheless, fetal arrhythmias could be concomitant with aneurysms of the oval fossa. Should that be the case, arrhythmias usually persist in childhood and postnatal life until an aneurysm resolves, ${ }^{15}$ unlike our clinical case.

Fetal arrhythmias should be evaluated by heart Doppler as well as cardiotocography, as an adjunct to biophysical profiles for fetal assessment. ${ }^{16}$

Special attention should be paid to trying to differentiate the outline of the thymus, to ensure an adequate delimitation between its echogenicity and that of the adjacent lung fields; this helps avoid confusion with anterior masses of the neck, such as goiter, cardiac masses such as rhabdomyoma, and pulmonary masses, such as cystic pulmonary airway malformation of the lung (CPAM). ${ }^{17}$ Should these differential diagnoses be ruled out, the patient can be reassured on physiological thymus hyperplasia.

\section{CONCLUSION}

The fetal thymus is a structure that usually goes unnoticed during the process of prenatal diagnosis, and when it presents alterations in its morphology, can lead to confusion.

\section{CLINICAL SIGNIFICANCE}

It is especially important to know the anatomical reference points and the regulated method for its measurement and thus be able to avoid, as far as possible, diagnostic errors that can lead to an increase in the state of anxiety to the patient and to the repetition or request of surplus diagnostic tests, with the consequent burden of unnecessary sanitary expense and patient's anxiety.

\section{REFERENCES}

1. Lele SM, Lele MS, Anderson VM. The thymus in infancy and childhood: embryologic, anatomic, and pathologic considerations. Chest Surg Clin N Am 2001;11:233-253.

2. Shimosato $Y$, Mukai K. Tumors of the thymus and related lesions. En: Shimosato Y, Mukai K, eds. Atlas of tumor pathology: tumors of the mediastinum, fasc. 21, ser. 3. Washington, DC: Armed Forces Institute of Pathology, 1997;158-168.

3. Zalel Y, Gamzu R, Mashiach S, et al. The development of the fetal thymus: an in utero sonographic evaluation. Prenat Diagn 2002;22.

4. Wacker-Gussmann A, Strasburger JF, Cuneo BF, et al. Fetal arrhythmias associated with cardiac rhabdomyomas. Heart Rhythm. 2014 Apr 1;11(4):677-683.

5. Dalla Costa M, Mangano FA, Betterle C. Thymic hyperplasia in patients with Graves' disease. Journal of endocrinological investigation. 2014 Dec 1;37(12):1175-1179.

6. Ryan AK, Goodship JA, Wilson DI, et al. Spectrum of clinical features associated with interstitial chromosome 22q 11 deletions: a European collaborative study. J Med Genet 1997;34:798-804.

7. Diemert A, Hartwig I, Pagenkemper M, et al. Fetal thymus size in human pregnancies reveals inverse association with regulatory $\mathrm{T}$ cell frequencies in cord blood. Journal of reproductive immunology. 2016 Feb 1;113:76-82.

8. Felker RE, Caetier MS, Emerson DS, et al. Ultrasound of the fetal thymus. J Ultrasound Med 1989;8:669-673.

9. Cho JY, Min JY, Lee YH, et al. Diameter of the normal fetal thymus on ultrasound. Ultrasound Obstet Gynecol. 2007;29(6).

10. De Leon-Luis J, Gámez F, Pintado P, et al. Sonographic measurements of the thymus in male and female fetuses. J Ultrasound Med. 2009;28:43-48.

11. Yildirim M, Ipek A, Dauletkazin G, et al. Sonographic measurement of the fetal thymus: Relationship with maternal obesity. Journal of Clinical Ultrasound. 2017 Jun;45(5):277-281. 
12. DeLeon-Luis J, Pintado P, Gamez F, et al.720: Ultrasonographic development of the thymus in fetus with Down syndrome: a prospective study. American Journal of Obstetrics and Gynecology [Internet]. Elsevier BV; 2009 Dec;201(6):S260.

13. Paladini D. How to identify the thymus in the fetus: the thybox. Ultrasound Obstet Gynecol. 2011;37:488-492.

14. Strasburger J, Cheulkar B, Wichman HJ. Perinatal Arrhythmias: Diagnosis and Management. Clin Perinatol. 2007;34(4): 627.
15. Rice MJ, McDonald RW, Reller MD. Fetal atrial septal aneurysm: A cause of fetal atrial arrhythmias. J Am Coll Caridiol 1988;12:1292-1297.

16. Wacker-Gussmann A, Cuneo B, Wakai R, Strasburger J. Diagnosis and Treatment of Fetal Arrhythmia. Am J Perinatol 2014;31:617-628.

17. Zafar HM, Ankola A, Coleman B. Ultrasound pitfalls and Artifacts Related to Six Common Fetal Findings. Ultrasound Quarterly 2012;28:105-124. 